\title{
Une histoire dans laquelle vivre
}

\section{Samia Hurst}

Institut Ethique Histoire Humanités (iEH2), Faculté de médecine, Genève

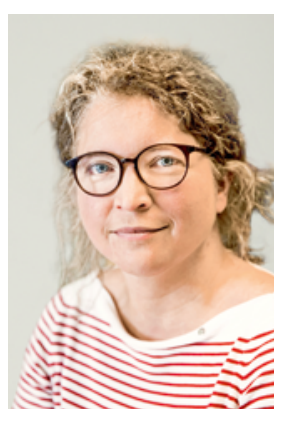

Et comment se débrouillait-il à la maison? Silence. Après deux mois d'hospitalisation et trois transferts, on avait oublié cette question. Nous racontons en permanence nos patients à nos collègues. Nous racontons leurs maladies, nos raisonnements, nos traitements, leurs réactions. Nous racontons beaucoup plus rarement qui ils sont et il arrive qu'on ne le sache plus. Cette scène est tirée de ces consultations d'éthique où personne n'est là pour raconter l'histoire de vie d'un malade et où l'on ne sait plus comment le soigner au mieux, parce qu'on ne sait plus qui il est.

Ce lien entre le récit et l'identité est loin d'être anodin. Nous nous racontons, très littéralement, notre vie. Ces récits nous constituent. La philosophe Hilde Lindemann va plus loin. Notre identité personnelle serait constituée de la pratique constante par laquelle nous tenons dans notre conscience, les uns pour les autres, des parts de notre histoire [1]. Notre identité est narrative: nous nous la racontons plutôt que de la comprendre. Nos proches, eux aussi, se racontent qui nous sommes: ils maintiennent ainsi une part de notre identité dans un processus qui commence avant la naissance et se poursuit après la mort. Nous vivons dans nos histoires, et c'est comme vivre dans un chantier perpétuel où chacun met en permanence la main à l'ouvrage.

\section{Nous vivons dans nos histoires, et c'est comme vivre dans un chantier perpétuel où chacun met en permanence la main à l'ouvrage.}

Etre SDF de ce domicile-là est une souffrance toute particulière. La maladie peut nous en priver, en faisant parfois complètement dérailler le récit. Cela peut faire partie de notre travail d'aider les personnes malades à retrouver une histoire dans laquelle vivre [2]. Lorsque nous disons que «ça n'a plus de sens» cela veut souvent dire qu'il n'est plus possible de trouver une histoire dans laquelle il soit possible d'habiter.

Alors que la maladie fragilise nos récits, il arrive malheureusement que le système de santé n'aide pas. Dans les services d'urgences, nous ôtons aux personnes les signes extérieurs de leur identité pour les mettre en «uniforme de patients». Lorsqu'elles nous racontent leur biographie, nous nous hâtons d'en venir à leurs symptômes. Lorsqu'elles se confient à nous, nous transmettons avec un soin moléculaire et presque affectueux tous les détails cliniques, mais souvent rien d'autre. Pourquoi tant de patients vivent-ils la maladie comme dépersonnalisante, alors que tant de professionnels sont bienveillants, éduqués, formés à la relation? En nous succédant devant eux et en gardant notre attention sur autre chose que leur histoire, nous ne contribuons pas à maintenir leur identité et parfois nous allons jusqu'à l'endommager.

\section{Il arrive que nos patient aient besoin d'une aide} active pour tenir en main leur biographie, face à la menace identitaire que peut constituer une maladie.

Cette erreur est profonde et elle est souvent due à une méconnaissance. Considérer nos patients comme des personnes à part entière est une évidence. Il arrive que nos patient aient besoin d'une aide active pour tenir en main leur biographie, face à la menace identitaire que peut constituer une maladie [3]. Et ça, c'est moins évident. C'est un chapitre où les progrès individuels sont là, mais où la complexité croissante des structures de la santé dresse aussi des obstacles plus grands que par le passé. Nous faisons mieux qu'avant, mais nous devrons faire mieux encore.

\section{Références}

1 Lindemann H. Holding and Letting Go: the Social Practice of Personal Identities. Oxford, New York: Oxford University Press; 2014

2 Charon R. Narrative medicine: form, function, and ethics. Annals of internal medicine. 2001 Jan 2;134(1):83-7. PubMed PMID: 11187429. Epub 2001/02/24.

3 Tavaglione N, Martin AK, Mezger N, Durieux-Paillard S, François A, Jackson Y, et al. Fleshing out vulnerability. Bioethics. 2015 Feb;29(2):98-107. PubMed PMID: 24602115. 